\title{
Anomaly analysis of brane-antibrane systems
}

\section{John H. Schwarz}

California Institute of Technology, Pasadena, CA 91125, USA

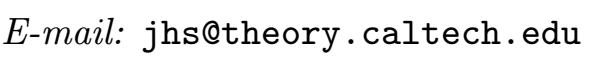

\section{Edward Witten}

Caltech-USC Center for Theoretical Physics

University of Southern California, Los Angeles, CA 90089, USA

E-mail: witênêias.edu

\begin{abstract}
String theories with branes can often be generalized by adding braneantibrane pairs. We explore the cancellation of anomalies in this more general context, extending the familiar anomaly-cancelling mechanisms, both for ten-dimensional string theories with D-branes and for certain supersymmetric compactifications.
\end{abstract}

Keywords: 'Duălity in Gauge Field Theories, Chern-Simons Theories, String' Diualityi. 


\section{Contents}

ii. Introduction

2. Type-IIB theory

12.1- The spectrum

2.2 Anomaly analysis

12.3 Inclusion of the tachyon field

3. Type-I theory

13.11 The spectrum

Microscopic derivation

Anomaly analysis

13.4 Theories with symplectic groups

44. Another class of ten-dimensional models

A. 11 The U(32) theory

Addition of brane-antibrane pairs

5. Type I compactified on a smooth K3

6. Type I compactified on a $\mathbb{T}^{4} / \mathbb{Z}_{2}$ orbifold

i6.1 Review of the basic model

The massless spectrum

16.3 Anomaly analysis

'6.4 Addition of brane-antibrane pairs

\section{Introduction}

Analysis of spacetime anomalies has played an important role in our understanding of string theories and branes. String theories with branes can be generalized by adding brane-antibrane pairs, including spacetime filling branes as in Ii]. In the present paper, we will analyze anomaly cancellation in this more general context, showing how the familiar mechanisms for anomaly cancellation can be generalized. We consider the type-IIB theory with D9-D $\overline{9}$ pairs in section $2_{-1}^{2}$ the type-I theory with

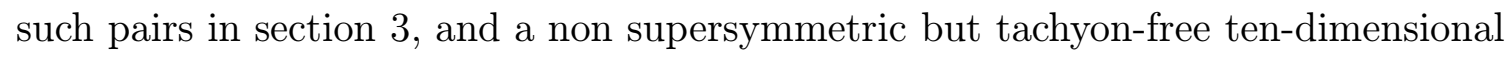




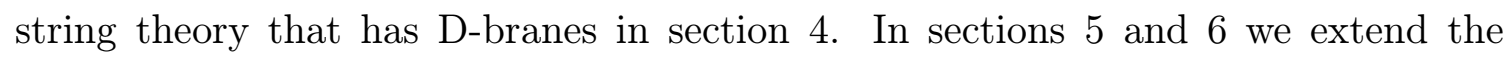
analysis to consider type-I compactifications on smooth K3 surfaces and on a simple K3 orbifold. In these examples, we consider D5-D $\overline{5}$ as well as D9-D $\overline{9}$ pairs.

\section{Type-IIB theory}

We begin by considering the type-IIB theory with added D9-branes and anti-D9branes. This system was considered first in [i]. In this section we will review the analysis of that paper filling in some of the details. Our reason for doing this is that we want to start with the simplest example of a chiral theory with added spacetime-filling brane-antibrane pairs in order to set the stage for the more complicated theories that we will discuss later. The anomaly cancellation mechanism that we will describe is closely related to the analysis considered in [i2] to determine certain effective couplings on branes. The main difference is that we focus on D9- and D $\overline{9}$-branes, while $\mathrm{D} p$ branes of $p<9$ were the focus of that paper. Type-II anomaly cancellation including

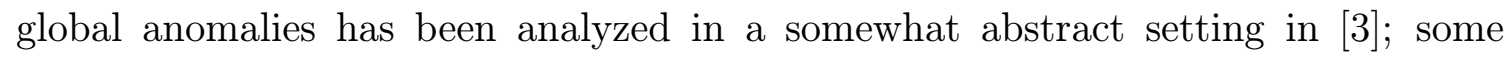
illustrative cases of the global anomalies were studied in [i卬⿱一𫝀].

Cancellation of Ramond-Ramond (R-R) tadpoles requires that the total D9brane charge should vanish. Therefore one must require that an equal number $n$ of D9-branes and anti-D9-branes are present.

\subsection{The spectrum}

The $n$ D9-branes have associated massless fields, arising as excitations of 99 openstrings, that correspond to a supersymmetric $\mathrm{U}(n)$ gauge theory. The adjoint Majorana-Weyl fermions have the same chirality (call it left-handed) as the gravitinos of the supergravity multiplet. The adjoint representation decomposes into irreducible representations with dimensions $n^{2}-1$ and 1 . The singlet fermion can be identified as the Goldstino associated to the supersymmetry broken by the presence of the branes. (See for the interpretation of the singlet massless fermion on a brane as a Goldstone fermion of spontaneously broken supersymmetry.) Similarly, the anti-D9-branes carry a second supersymmetric $\mathrm{U}(n)$ gauge theory. Its fermions have the same chirality (left-handed) as those of the branes. The singlet fermion of the anti-branes is the Goldstino associated to breaking of the other supersymmetry of the IIB theory. So, when both the branes and anti-branes are present, the supersymmetry is completely broken. The fact that they each carry fermions of the same chirality is clearly required, since the two supersymmetries of the IIB theory have the same chirality. Altogether, the combined system is a non-supersymmetric theory with $\mathrm{U}(n) \times \mathrm{U}(n)$ gauge symmetry.

Such a system is unstable, of course, since the branes and anti-branes will tend to annihilate. This is reflected in the presence of tachyons in the spectrum. Specifically, the oriented type-IIB open strings that connect the branes to the anti-branes 
have the opposite GSO projection from those that connect branes to branes or antibranes to anti-branes. As a result, the spectrum contains tachyons $T$ in the bifundamental representation $(n, \bar{n})$. These scalar fields are complex, of course. In addition to the tachyons, the open strings connecting branes to anti-branes also give massless fermions in the bifundamental representation. These fermions have the opposite chirality (right-handed) to the other ones as a consequence of the opposite GSO projection.

\subsection{Anomaly analysis}

Even though the brane-antibrane system in question is unstable, it should make sense within the context of perturbation theory. The point where the tachyon field vanishes $(T=0)$ corresponds to a local maximum of the tachyon potential, and thus it is part of a classical solution. The one-loop effective action in an expansion around this solution should be well defined, even though the solution is unstable, and in particular it should have a well-defined phase. Therefore, the various gauge and gravitational anomalies, which arise as one-loop effects, should cancel. The chiral fields of the type-IIB supergravity multiplet give cancelling contributions to the gravitational anomalies, just as they do in the absence of the D-branes [6]. However, there are now additional chiral fermions in the spectrum, which also contribute to anomalies. So that needs to be analyzed. We will begin by setting $T=0$, but later we will incorporate the dependence on $T$.

Let us use subscripts 1 and 2 to refer to the two $\mathrm{U}(n)$ groups. Then the anomaly contributed by the chiral fermions associated to the branes is characterized by the 12-form part of the following expression:

$$
I=\left(\operatorname{Tr} e^{i F_{1}}+\operatorname{Tr} e^{i F_{2}}-\operatorname{tr} e^{i F_{1}} \operatorname{tr} e^{-i F_{2}}-\operatorname{tr} e^{-i F_{1}} \operatorname{tr} e^{i F_{2}}\right) \hat{A}(R) .
$$

Here $\hat{A}(R)$ is the Dirac index. The symbol Tr refers to the adjoint representation, whereas the symbol tr refers to the fundamental representation. The coefficients +1 are introduced for the left-handed Majorana-Weyl fermions, whereas the coefficient -1 appears for right-handed Weyl fermions and their complex conjugates.

The adjoint representation of $\mathrm{U}(n)$ is given by the product $n \times \bar{n}$. As a result, using a basic property of the Chern character, we have

$$
\operatorname{Tr} e^{i F}=\operatorname{tr} e^{i F} \operatorname{tr} e^{-i F} .
$$

This enables us to recast eq. (2.2. $\left.\overline{1}^{\prime}\right)$ in the form $I=Y \bar{Y}$, where

$$
Y=\left(\operatorname{tr} e^{i F_{1}}-\operatorname{tr} e^{i F_{2}}\right) \sqrt{\hat{A}(R)}
$$

and

$$
\bar{Y}=\left(\operatorname{tr} e^{-i F_{1}}-\operatorname{tr} e^{-i F_{2}}\right) \sqrt{\hat{A}(R)} .
$$

The anomaly polynomial $I_{12}$ is not zero, but because of the factorization $I=Y \bar{Y}$, the

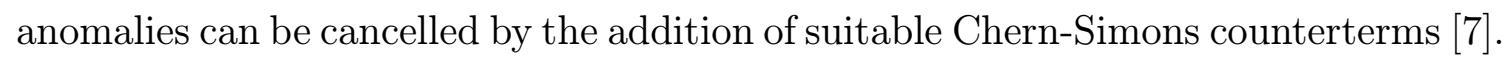


The determination of the anomaly cancelling counterterms has some (inconsequential) ambiguities, which we will resolve by making the most symmetrical choice. This will give the most elegant formulas, and it will make contact with previous results in the literature. Now let us define $\Omega$ by $Y=d \Omega$, where it is understood that Chern-Simons terms are introduced for the gauge field factor leaving the factor $\sqrt{\hat{A}(R)}$ in tact. This is desirable because $\sqrt{\hat{A}(R)}$ has a constant term, whereas the constant terms arising from the gauge field factor cancel. Let us denote the variation of $\Omega$ under local gauge transformations by $\delta \Omega=d \Lambda$. The anomaly can now be cancelled by introducing the non-trivial gauge transformation rules for the R-R fields $C=-i C_{0}+C_{2}+i C_{4}-C_{6}-i C_{8}$

$$
\delta C=\Lambda \text {. }
$$

It follows that the gauge-invariant $\mathrm{R}-\mathrm{R}$ field strengths are given by $H=d C-\Omega$. In particular, this implies that the $\mathrm{R}-\mathrm{R}$ scalar $C_{0}$ is eaten by the relative $\mathrm{U}(1)$ gauge field $\operatorname{tr} A_{1}-\operatorname{tr} A_{2}$, which then becomes massive. We also note the Bianchi identity $d H+Y=0$.

Now we can write down the anomaly-cancelling Chern-Simons term in the form

$$
S_{\mathrm{CS}}=\mu \int C Y
$$

where $\mu$ is a normalization constant. This expression is real with the phase choices made in the definition of $C$. The fact that R-R fields other than $C_{2}$ can be involved in anomaly cancellation was first recognized in [8]. An interpretation of the Chern-

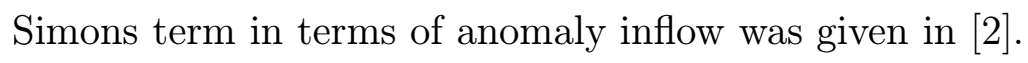

In writing the couplings, we have ignored the self-duality of the R-R fields. One standard approach to dealing with this is to treat half of the $C$ 's as independent fields, in which case half of the anomaly-canceling couplings remain as we have written them and the other half become contributions to the Bianchi identities. The self-duality of the "middle" R-R field $G_{5}=d C_{4}$ makes this procedure subtle to interpret for type IIB. There are various procedures for dealing with this, and we will not discuss the issue here. A more abstract way of interpreting actions with self-dual R-R fields is

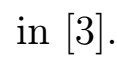

\subsection{Inclusion of the tachyon field}

A natural generalization of the Chern-Simons action to include the dependence on

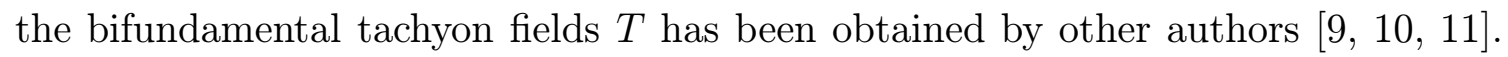
The result can be elegantly described as replacing the factor $\left(\operatorname{tr} e^{i F_{1}}-\operatorname{tr} e^{i F_{2}}\right)$, which appears in the expression (2.2.3 that has been called the superconnection [12]. The curvature of the superconnection is given by the $2 n \times 2 n$ matrix

$$
i \mathcal{F}=\left(\begin{array}{cc}
i F_{1}-T T^{\dagger} & D T^{\dagger} \\
D T & i F_{2}-T^{\dagger} T
\end{array}\right)
$$


The supertrace Str is the difference of the traces of the upper left block and the lower right block, as usual. Clearly, Str $e^{i \mathcal{F}}$ reduces to our previous expression for $T=0$. In fact, this notation is convenient even in that case.

If the tachyon field can be considered large, then $\operatorname{Str} e^{i \mathcal{F}}$ is very small except near zeroes of $T$ - or at least points at which some eigenvalues of $T$ are zero. Indeed, in this situation the diagonal elements of $e^{i \mathcal{F}}$ are proportional to $e^{-T T^{\dagger}}$ and $e^{-T^{\dagger} T}$, which can be considered small. Note that in the vacuum, $T$ is a unitary matrix times a fixed constant, so $T T^{\dagger}$ and $T^{\dagger} T$ are equal and are multiples of the identity. So if we can ignore $F_{1}, F_{2}$, and $D T$, then $\operatorname{Str} e^{i \mathcal{F}}$ vanishes pointwise. In practice, an important case is that on the complement of a submanifold that is interpreted as a $p$-brane world-volume for some $p<9, T$ has its vacuum expectation value (up to a gauge transformation). Such a tachyon field describes [i] $\left.\overline{1}_{1}\right]$ D9-D $\overline{9}$ annihilation to a $\mathrm{D} p$-brane. If all length scales in the problem are very large compared to the string scale, then in the usual generalized "vortex" configuration describing tachyon condensation, $F_{1}, F_{2}$, and $D T$ all vanish exponentially fast with the distance from the $\mathrm{D} p$-brane. So in this limit, the anomalous couplings are given by a differential form that has its support on the $\mathrm{D} p$-brane, rather than on the 9-branes where we started.

Superconnections were introduced in the first place [i] $\overline{2}_{-1}$ precisely to give such an analytic proof of "localization" of various topological quantities (such as the anomalies of interest to us here) as well as to explain various physical results. From the point of view of the application to D9-D $\overline{9}$ annihilation, the fact that this works out correctly is one consistency check on the claim that a D9-D $\overline{9}$ system with no net D9-brane charge and carrying suitable gauge fields can annihilate to a $\mathrm{D} p$-brane with $p<9$. This check of the tachyon condensation story is not really independent of analyses in the previous literature, but it is perhaps an interesting way to look at things.

\section{Type-I theory}

We now wish to repeat the analysis of the preceding section for the type-I theory. Recall that this theory has an orientifold plane carrying -32 units of R-R charge, which is cancelled by the contribution of 32 D9-branes. The D9-branes give SO(32) gauge symmetry. They do not break any supersymmetry, and accordingly the open string spectrum contains no massless Goldstinos. The various local anomalies cancel as a result of a well-known analysis analogous to that of the preceding section $\left[\bar{T}_{1}\right]$. We now wish to generalize this setup to include $n$ additional D9-brane anti-D9-brane pairs. This system has been considered previously by Sugimoto in [1] 1 in. Our purpose in reviewing these results is to fill in some details and to set the stage for more complicated examples. 


\subsection{The spectrum}

The gauge group of the $32+n$ D9-branes is expected to be $\mathrm{SO}(32+n)$, and that of the $n$ anti-D9-branes is expected to be $\mathrm{SO}(n)$. Given our knowledge of the $n=0$ case, it is pretty clear that the D9-branes should give a supersymmetric $\mathrm{SO}(32+n)$ gauge theory. Thus the 99 open strings should give massless gauge bosons and massless left-handed MW fermions, each in the adjoint representation. As before, the $9 \overline{9}$ open strings have the opposite GSO projection. Therefore they give real tachyon fields in the bifundamental representation $(32+n, n)$ and right-handed MW fermions that are also in the bifundamental representation $(32+n, n)$.

This still leaves the question of the spectrum of $\overline{9} \overline{9}$ open strings. Momentarily we will sketch how to deduce this spectrum from first principles. For the moment, we simply look for a choice that satisfies some reasonable expectations. In this problem, we do not expect any tachyons, but we do expect massless vector fields in the adjoint of $\mathrm{SO}(n)$. We expect the anti-D9-branes to break the supersymmetry, and therefore there should be a massless left-handed MW Goldstino, which is a singlet of the gauge group. Since there is no singlet gauge boson this tells us that the antiD-brane spectrum cannot be supersymmetric. That is okay - there is no reason that it should be. To figure out what other fermions are required, let us consider the minimal requirement of anomaly cancellation - namely, the cancellation of the $\operatorname{tr} R^{6}$ term. This tells us that the branes and the anti-branes should contribute a net of 496 left-handed MW fermions. We already have $(32+n)(31+n) / 2$ from the 99 open strings. From this we must subtract $n(32+n)$ to take account of the right-handed MW fermions associated to the $9 \overline{9}$ open strings. Therefore, to end up with 496 , the $\overline{9} \overline{9}$ open strings need to contribute a net of $n(n+1) / 2$ left-handed MW fermions. The obvious way to achieve this is with the sum of a symmetric traceless tensor and a singlet. ${ }^{1}$ It is very gratifying that there is a singlet, since it is the required Goldstino.

\subsection{Microscopic derivation}

Now we will give a microscopic derivation of the spectrum.

One way to describe a system with D-antibranes as well as D-branes is to assign variable statistics to the Chan-Paton states [1] $\left.{ }_{-1}^{-}\right]$. Let us briefly describe how this works. Consider a system of D-branes with a Chan-Paton label that takes $p+q$ values; take the first $p$ states to be bosonic and the last $q$ to be fermionic. By a bosonic state we mean a state on which the GSO operator $(-1)^{F}$ has eigenvalue +1 , while on a fermionic state it has eigenvalue -1 . We claim that such a system describes a collection of $p$ D-branes and $q$ D-antibranes.

To verify this, we examine the spectrum, considering first type-II superstrings (so that the open strings are oriented). The D-D strings are states both of whose ends have bosonic Chan-Paton labels. $(-1)^{F}$ acts by +1 on the Chan-Paton wavefunction

\footnotetext{
${ }^{1}$ This result is related by T-duality to an analogous result for D2-branes obtained in [1든.
} 
of such a string, so for a state to have $(-1)^{F}=1$ overall, the internal part of the wavefunction (made from the string oscillators) must also have $(-1)^{F}=1$. This leads to the standard GSO projection, giving a spectrum with a massless gauge field and no tachyon. Likewise, a $\overline{\mathrm{D}}-\overline{\mathrm{D}}$ string has a fermionic label at each end, so $(-1)^{F}$ acts on the Chan-Paton wavefunction by $(-1)^{2}=+1$, and again the $\overline{\mathrm{D}}-\overline{\mathrm{D}}$ strings have the conventional GSO projection. But for $\mathrm{D}-\overline{\mathrm{D}}$ strings, the result is different. In this case, there is a bosonic label at one end of the string and a fermionic one at the other end, so the Chan-Paton wavefunction has $(-1)^{F}=-1$. Hence to get an overall value $(-1)^{F}=1$, the internal wavefunction must likewise have $(-1)^{F}=-1$. The projection onto these states is the opposite of the usual GSO projection, and it gives for the $\mathrm{D}-\overline{\mathrm{D}}$ strings a spectrum with a tachyon and no massless gauge field, and opposite chirality for massless fermions in the Ramond (R) sector. These are of course the standard results for type II.

We now move on to the case of type-I superstrings with $p$ D9 and $q$ D $\overline{9}$ branes. Again we represent this system by allowing $p$ bosonic and $q$ fermionic labels for the Chan-Paton factors. (For tadpole cancellation, one eventually wants $p-q=32$.) The novel ingredient is that the open strings are unoriented; we must define an operator $\Omega$ that exchanges the two ends of an open string, and project onto states with $\Omega=1$. For D9-D9 strings in type I, the $\Omega$ projector is the usual one that leaves an $\mathrm{SO}(p)$ gauge group. (If we take the opposite projector, we get instead the theory discussed in section 's. $\overline{3}$ ' below.) For D9-D $\overline{9}$ and D $\overline{9}$-D9 strings, the $\Omega$ projector simply identifies the D9-D $\overline{9}$ and D $\overline{9}-\mathrm{D} 9$ states, so the correct choice of $\Omega$ projector is needed to find the correct wavefunctions but not to find the spectrum. What about the $\mathrm{D} \overline{9}-\mathrm{D} \overline{9}$ strings? In this case, we want to show that the $\Omega$ projector is the usual one in the Neveu-Schwarz (NS) sector, but is opposite to the usual one in the R sector. If that is true, the Chan-Paton wavefunctions for massless D $\overline{9}-\mathrm{D} \overline{9}$ strings in the NS sector will be the usual antisymmetric tensors $\psi_{i j}=-\psi_{j i}, i, j=1, \ldots, q$, leading to massless $\mathrm{SO}(q)$ gauge fields. But the Chan-Paton wave functions of massless D $\overline{9}-\mathrm{D} \overline{9}$ strings in the $\mathrm{R}$ sector will be symmetric tensors $\chi_{i j}=\chi_{j i}$, which is the spectrum of left-handed massless MW fermions from D $\overline{9}-\mathrm{D} \overline{9}$ strings that was claimed above.

To reduce the question to a standard one, note that defining the correct $\Omega$ operator is equivalent to knowing how to compute the Moebius strip contribution to the open string partition function. This is for a familiar reason: a Moebius strip worldsheet can arise from a trace in the open string Hilbert space with a factor of $\Omega$ inserted. Now the Moebius strip has only a single boundary. Let us represent the Moebius strip in the standard fashion as the strip $0 \leq \sigma^{1} \leq \pi,-\infty<\sigma^{2}<\infty$ in the $\sigma^{1}-\sigma^{2}$ plane, with the equivalence

$$
\sigma^{1} \longrightarrow \pi-\sigma^{1}, \quad \sigma^{2} \longrightarrow \sigma^{2}+2 \pi t
$$

The Moebius strip has only one boundary component, a circle $C$ that we can iden- 
tify as $\sigma^{1}=0,0 \leq \sigma^{2} \leq 4 \pi t$. A path integral on the Moebius strip computes $\operatorname{Tr}(-1)^{\beta F} \Omega e^{-2 \pi t H}$, where $\beta \in 0,1$ determines the spin structure in the $\sigma^{2}$ direction and $F$ is the number of worldsheet fermions.

If the Chan-Paton labels are bosonic, then $p$ Chan-Paton states propagating around $C$ give a factor of $p$ in the path integral, multiplying the usual evaluation of $\operatorname{Tr}(-1)^{\beta F} \Omega e^{-2 \pi t H}$ by $p$ and leaving unchanged the usual $\Omega$ projection. But $q$ fermionic Chan-Paton states propagating around $C$ give a factor of $+q$ if the spin structure restricted to $C$ is in the NS sector, and $-q$ if the spin structure restricted to $C$ is in the $\mathrm{R}$ sector. ${ }^{2}$ If the Chan-Paton states contribute a factor of $q$ to $\operatorname{Tr}(-1)^{\beta F} \Omega e^{-2 \pi t H}$, this corresponds to the usual $\Omega$ projection, but if they contribute a factor $-q$, we interpret this in an operator language to mean that the sign of $\Omega$ is reversed, and we get the opposite-to-usual $\Omega$ projection.

The path integral on the Moebius strip, viewed in the open string channel as computing $\operatorname{Tr}(-1)^{F} \Omega e^{-2 \pi H}$, receives contributions from both the NS and R sectors of the open string. A standard result, explained on [i] ${ }_{1}^{1}$, page 42], is that the spin structure on $C$ is actually the same as that of the open strings. Thus, looking at the Moebius strip in the closed string channel, a closed string wrapped once around $C$ is in the NS-NS sector if the open strings are in the NS sector, and in the R-R sector if the open strings are in the $\mathrm{R}$ sector. (In particular, the spin structure on $C$ does not depend on whether the worldsheet fermions are periodic or antiperiodic under $\sigma^{1} \rightarrow \pi-\sigma^{1}, \sigma^{2} \rightarrow \sigma^{2}+2 \pi t$. That is because $C$ covers the range $0 \leq \sigma^{2} \leq 4 \pi t$ and so wraps twice around the $\sigma^{2}$ direction.)

Applied to our problem, the statement about the spin structure on $C$ means that, as we have claimed above, the open strings in the NS sector have the standard $\Omega$ projection, and those in the $\mathrm{R}$ sector have the opposite projection. For completeness, we summarize how the claim about the spin structure on $C$ is established. The transformation $\sigma^{1} \rightarrow \pi-\tilde{\sigma}^{1}, \sigma^{2} \rightarrow \sigma^{2}+2 \pi t$, exchanges left- and right-moving worldsheet fermions $\psi$ and $\widetilde{\psi}$, so under this transformation

$$
\psi\left(\sigma^{1}, \sigma^{2}+2 \pi t\right)=-(-1)^{\beta} \widetilde{\psi}\left(\pi-\sigma^{1}, \sigma^{2}\right),
$$

where $\beta \in\{0,1\}$ determines the spin structure of the Möbius strip in the $\sigma^{2}$ direction. It is convenient to combine $\psi$ and $\widetilde{\psi}$ to a function defined for all values of $\sigma^{1}$; we extend $\psi$ to $\sigma^{1}<0$ by setting $\psi\left(-\sigma^{1}, \sigma^{2}\right)=\widetilde{\psi}\left(\sigma^{1}, \sigma^{2}\right)$, and to $\sigma^{1}>\pi$ by setting $\psi\left(\sigma^{1}, \sigma^{2}\right)=(-1)^{\alpha} \psi\left(\pi-\sigma^{1}, \sigma^{2}\right)$, where $\alpha \in 0,1$, with $\alpha=0$ for the $\mathrm{R}$ sector of open strings and $\alpha=1$ for the NS sector. Altogether $\psi\left(\sigma^{1}, \sigma^{2}\right)$ is naturally extended to a field periodic in $\sigma^{1}$ with

$$
\psi\left(\sigma^{1}+2 \pi, \sigma^{2}\right)=(-1)^{\alpha} \psi\left(\sigma^{1}, \sigma^{2}\right) .
$$

\footnotetext{
${ }^{2}$ In other words, we get $+q$ if worldsheet fermions restricted to $C$ are antiperiodic and $-q$ if they are periodic. In the former case, the path integral has a factor which is a trace in the Chan-Paton Hilbert space, to which all states contribute +1 , while in the latter case the trace is replaced by $\operatorname{Tr}(-1)^{F}$, and fermionic states contribute -1 .
} 
In terms of the extended field $\psi,\left(\begin{array}{l}\overline{3} \\ \overline{3}\end{array} \overline{2}-\overline{1}\right)$ can be written

$$
\psi\left(\sigma^{1}, \sigma^{2}+2 \pi t\right)=-(-1)^{\beta} \psi\left(\sigma^{1}-\pi, \sigma^{2}\right)
$$

By applying this relation twice, we get

$$
\psi\left(\sigma^{1}, \sigma^{2}+4 \pi t\right)=\psi\left(\sigma^{1}-2 \pi, \sigma^{2}\right)=(-1)^{\alpha} \psi\left(\sigma^{1}, \sigma^{2}\right) .
$$

So the worldsheet fermions at $\sigma^{1}=0$ transform under $\sigma^{2} \rightarrow \sigma^{2}+4 \pi$ by $(-1)^{\alpha}$, and hence the spin structure on $C$ is the same as the spin structure in the open string channel at $\sigma^{2}=0$, as was to be shown.

\subsection{Anomaly analysis}

Having identified the spectrum, we can now analyze the anomalies. We have already arranged for the cancellation of the $\operatorname{tr} R^{6}$ term, but there is quite a bit more that needs to be checked. The formulas must reduce to the standard ones of the type-I theory for $n=0$, so it is really the $n$ dependent terms that are in issue. We will let the index 1 refer to the $\mathrm{SO}(32+n)$ group and the index 2 refer to the $\mathrm{SO}(n)$ group. By standard manipulations, one finds that just as in the $n=0$ case the total anomaly 12-form factorizes into a product of a four-form and an eight-form, $I_{12}=2 Y_{4} Y_{8}$, where

$$
Y_{4}=\frac{1}{2}\left(\operatorname{tr} R^{2}-\operatorname{tr} F_{1}^{2}+\operatorname{tr} F_{2}^{2}\right)
$$

and

$$
Y_{8}=\frac{1}{24}\left(\frac{1}{8} \operatorname{tr} R^{4}+\frac{1}{32}\left(\operatorname{tr} R^{2}\right)^{2}+\left(\operatorname{tr} F_{1}^{4}-\operatorname{tr} F_{2}^{4}\right)-\frac{1}{8} \operatorname{tr} R^{2}\left(\operatorname{tr} F_{1}^{2}-\operatorname{tr} F_{2}^{2}\right)\right),
$$

where the subscript 1 refers to $\mathrm{SO}(n+32)$ and the subscript 2 refers to $\mathrm{SO}(n)$. This factorization assures us that there is a Chern-Simons counterterm for which the anomaly cancels, just as in the $n=0$ case.

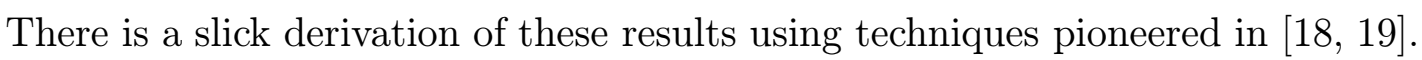
(See also [20-0] open strings give left-handed Majorana-Weyl fermions in the antisymmetric tensor representation $(\mathrm{A})$ of $\mathrm{SO}(32+n)$, the $\overline{9} \overline{9}$ open strings give left-handed MajoranaWeyl fermions in the symmetric tensor representation (S) of $\mathrm{SO}(n)$, and the $9 \overline{9}$ open strings give right-handed Majorana-Weyl fermions in the bifundamental representation. Thus the anomaly polynomial $I_{12}$ is proportional to the 12 -form piece of

$$
\frac{1}{2}\left(\operatorname{Tr}_{A} e^{i F_{1}}+\operatorname{Tr}_{S} e^{i F_{2}}-\operatorname{tr} e^{i F_{1}} \operatorname{tr} e^{i F_{2}}\right) \hat{A}(R)+\frac{1}{16} L(R) .
$$

The last term is the contribution of the gravitino and the dilatino from the closed- 
string sector. It is expressed here as half of the contribution of a self-dual R-R field. ( $L(R)$ is the Hirzebruch L-polynomial.) The reason this is correct is that the anomaly cancellation of the type-IIB theory implies that the contribution of a self-dual R-R field cancels that of two Majorana-Weyl gravitinos and dilatinos.

Using the identities

$$
\operatorname{Tr}_{A} e^{i F}=\frac{1}{2}\left(\left[\operatorname{tr} e^{i F}\right]^{2}-\operatorname{tr} e^{2 i F}\right)
$$

and

$$
\operatorname{Tr}_{S} e^{i F}=\frac{1}{2}\left(\left[\operatorname{tr} e^{i F}\right]^{2}+\operatorname{tr} e^{2 i F}\right),
$$

the expression (13.8. $)$ can be recast in the form

$$
\frac{1}{4}\left(\left[\operatorname{Str} e^{i F}\right]^{2}-\operatorname{Str} e^{2 i F}\right) \hat{A}(R)+\frac{1}{16} L(R),
$$

where $\operatorname{Str} e^{i F}=\operatorname{tr} e^{i F_{1}}-\operatorname{tr} e^{i F_{2}}$. Remarkably, the 12-form piece of this expression agrees with the 12 -form piece of $\frac{1}{4} Y^{2}$, where $Y$ is given by

$$
Y=\operatorname{Str} e^{i F} \sqrt{\hat{A}(R)}-32 \sqrt{L\left(\frac{R}{4}\right)} .
$$

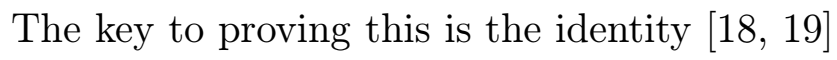

$$
\sqrt{\hat{A}(R) L(R / 4)}=\hat{A}\left(\frac{R}{2}\right) .
$$

This identity is an immediate consequence of the defining relations

$$
\hat{A}(R)=\prod_{i} \frac{\lambda_{i} / 2}{\sinh \lambda_{i} / 2}
$$

and

$$
L(R)=\prod_{i} \frac{\lambda_{i}}{\tanh \lambda_{i}} .
$$

In writing the anomaly 12 -form as $Y^{2} / 4$, one also needs to know that the 12 -form part of $\operatorname{Str} e^{i F} \hat{A}(R / 2)$ is the same as the 12 -form part of $2^{-6} \operatorname{Str} e^{2 i F} \hat{A}(R)$, since that 12 -form is homogeneous of degree 6 in $F$ and $R$.

Thus, just as in the IIB case, the anomaly $I_{12}$ is proportional to the 12 -form part of $I=Y^{2}$. Since we take the net D9-brane charge to be 32, the 0-form part of $Y=Y_{0}+Y_{4}+Y_{8}+\cdots$ vanishes, so the characteristic classes $Y_{4}$ and $Y_{8}$ are the two leading terms in the expansion of $Y$, and $I_{12}$ is proportional to $Y_{4} Y_{8}$.

The first term in the expression ( (or world-sheet boundary) contribution, whereas the second term is the orientifold plane (or cross-cap) contribution. The zero-form piece of $Y$ cancels between the two terms. This is the cancellation of the R-R tadpole. The three terms in $Y^{2}$ correspond to the contributions of the annulus, the Moebius strip, and the Klein bottle. 
The original study of the type-I anomaly utilized the R-R 2-form $C_{2}$ but not the dual R-R 6-form $C_{6}$. In order to give a symmetrical (but informal) treatment, analogous to that described for the IIB theory in the previous section, we will include both fields in the discussion that follows. Accordingly we define $C=C_{2}-C_{6}$. This enables us to proceed exactly as in the IIB case. Specifically, we again introduce

$$
S_{\mathrm{CS}}=\mu \int C Y
$$

where $\mu$ is a normalization constant. Then we define $\Omega$ by $Y=d \Omega$ and denote the variation of $\Omega$ under local gauge and Lorentz transformations by $\delta \Omega=d \Lambda$. Since only the four-form and eight-form pieces of $Y$ contribute, only the two-form and six-form pieces of $\Lambda$ are relevant. It then follows that the anomaly cancels for

$$
\delta C=\Lambda
$$

and that the gauge-invariant R-R field strengths are given by $H=d C-\Omega$.

It is natural to suppose that the tachyon dependence could be added in the same way as in the type-IIB theory. Specifically, in the formula for $Y$ we would replace $\operatorname{tr} e^{i F_{1}}-\operatorname{tr} e^{i F_{2}}$ by $\operatorname{Str} e^{i \mathcal{F}}$, where now the curvature of the superconnection is the $(2 n+32) \times(2 n+32)$ matrix

$$
i \mathcal{F}=\left(\begin{array}{cc}
i F_{1}-T T^{t} & D T^{t} \\
D T & i F_{2}-T^{t} T
\end{array}\right)
$$

since $T$ is real. The relation to brane annihilation is the same as before.

\subsection{Theories with symplectic groups}

It was noted in ${ }_{1}^{1}+\overline{4}$ that it is possible to construct a tachyon-free non-supersymmetric theory by modifying the orientifold projection that is used in constructing the type-I theory out of the type-IIB theory. Specifically, instead of modding out by $\Omega$ - the usual $Z_{2}$ symmetry, discussed in section '3.2.2', that is utilized in the type-I construction — one mods out by the $Z_{2}$ symmetry $\bar{\Omega}^{\prime}=\Omega(-1)^{F_{L}}$, where $F_{L}$ is the world-sheet fermion number for left-movers. The resulting theory contains an orientifold plane which has $\mathrm{R}-\mathrm{R}$ charge +32 , which is the opposite sign from the usual case. Accordingly, it is necessary to add 32 anti-D9-branes to cancel the R-R charge. Moreover, in this situation the gauge group associated to the anti-D9-branes is the symplectic group that is variously called $\operatorname{Sp}(16)$ or $\operatorname{USp}(32)$.

This theory is tachyon-free, but it contains a tree-level dilaton potential term. The orientifold plane has positive tension (as well as positive charge). Thus, in contrast to the usual type-I theory, this tension reinforces the tension of the D9-branes, to give a total vacuum energy of $64 T_{D 9}$ i 14 . In string metric this includes a factor of $e^{-\phi}$, where $\phi$ represents the dilaton. In canonical metric this becomes $e^{3 \phi / 2}$. 
Therefore, the runaway dilaton would appear to drive the system to zero coupling. It may be possible to achieve stability and finite coupling with a warped compactification $[\overline{2} \overline{2} \overline{1}, \overline{2} \overline{2} \overline{2}]$. At any rate, a weak-coupling anomaly analysis in ten-dimensional Minkowski space should make sense.

The closed string spectrum (at zero coupling) is identical to that of the type-I theory. In particular, it contains a massless gravitino. This is somewhat surprising, since the open string sector associated to the anti-D9-branes is not supersymmetric. In addition to the $\mathrm{Sp}(16)$ gauge bosons, the massless spectrum contains MajoranaWeyl fermions in the antisymmetric tensor representation of the gauge group. In contrast to the case of orthogonal groups, this multiplet is not the adjoint, which is a symmetric tensor. In fact, it is reducible into a part that has no symplectic trace and a singlet. The singlet can be identified as the Goldstino associated with the broken supersymmetry. Both the gravitino and the Goldstino have only one chirality, a situation that would be impossible in a maximally symmetric ten-dimensional spacetime (such as Minkowski or de Sitter space), for supersymmetry breaking in such a spacetime would require the gravitino to get mass, and this is only possible if both chiralities are present. Supersymmetric invariance of a theory with such an inedible Goldstino, which has only one chirality and cannot combine with the graviton to give a massive state, depends on the dilaton potential term in the effective action, as was demonstrated in [2] 3 . Because this term is present, such a theory does not lead to a maximally symmetric ten-dimensional spacetime, and the chirality of the gravitino and Goldstino leads to no contradiction.

Even though this theory has many differences from the type-I theory, the anomaly analysis works in exactly the same way. The antisymmetric tensor representation of $\operatorname{Sp}(16)$ has dimension 496, just as in the $\mathrm{SO}(32)$ case. Moreover, all of its charges with respect to the maximal torus (Cartan subalgebra) are identical to those in the $\mathrm{SO}(32)$ case. Therefore, since the massless closed string sector is identical to that of the type-I theory, the anomaly analysis is the same. Moreover, as discussed in [1] 1 in , one can also add additional brane antibrane pairs to make an unstable theory with $\operatorname{Sp}(16+n) \times \operatorname{Sp}(n)$ gauge symmetry. The anomaly analysis of this system is identical to that of the $\mathrm{SO}(32+2 n) \times \mathrm{SO}(2 n)$ theory described in the previous section.

\section{Another class of ten-dimensional models}

Three examples of ten-dimensional tachyon-free string theories without spacetime supersymmetry are known. The first one discovered is the $\mathrm{SO}(16) \times \mathrm{SO}(16)$ heterotic theory [24, 125. lend itself to the type of analysis we are doing here. A second example is the $\operatorname{Sp}(16)$ theory discussed in the previous section. The third example, which is the subject of this section, has $\mathrm{U}(32)$ gauge symmetry. It was discovered by Sagnotti in $\left[\overline{2} \overline{6}, \overline{2}, \overline{2} \overline{\eta_{1}}\right]$. 


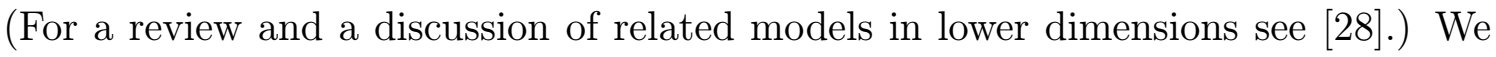
will quickly recall the essential features of this theory and then consider including additional brane antibrane pairs to give a theory with $\mathrm{U}(32+n) \times \mathrm{U}(n)$ gauge symmetry. Other examples of tachyon-free models without supersymmetry, which we will not consider here, have been constructed in lower dimensions [2],

\subsection{The U(32) theory}

A variant of the type-IIB superstring theory, usually called the type-0B theory, is constructed by making a different GSO projection from the usual one [2 $2 \overline{4}, 131]$. It also gives a modular invariant partition function and therefore is perturbatively consistent. The type-0B theory is a theory of oriented closed strings only. It involves no orientifold plane or spacetime filling D-branes. The spectrum includes the closed string tachyon, which is ordinarily removed by the GSO projection. The entire spectrum contains bosons only, since the R-NS and NS-R sectors are both projected out. At the massless level, the NS-NS spectrum is the same as for type-II superstrings: a graviton, two-form, and dilaton. The massless R-R spectrum is double that of the IIB theory. By this we mean that there is no self-duality constraint on the R-R spectrum. Evidently, the $0 \mathrm{~B}$ theory is non chiral, and so it is trivially anomaly-free.

Sagnotti's U(32) theory is constructed as an orientifold projection of the type-0B theory. The resulting theory has unoriented breakable strings, rather like the type-I theory. The orientifold projection removes the tachyon and half the massless R-R fields from the spectrum. The remaining massless $\mathrm{R}-\mathrm{R}$ fields are identical to those of the IIB theory. The massless NS-NS spectrum consists only of the graviton and the dilaton, just as in the type-I theory. There are still no fermions in the closed string spectrum. Thus the closed string spectrum includes just one chiral field: the four-form R-R potential whose field strength is self-dual. Clearly, if this were the whole story, the theory would be anomalous. However, there is an orientifold plane that carries -32 units of R-R charge (just as in the type-I theory) which requires the addition of 32 spacetime-filling D9-branes. Properties of some of the other D-branes in this theory have been discussed in $[5 \overline{2}, 153 \overline{3}$.

As was explained by Sagnotti, and will be clear from the anomaly analysis, the massless open-string spectrum contains U(32) gauge fields. It also contains Weyl fermions that belong to the antisymmetric tensor representation (496) of the gauge group. Sagnotti showed that these fermions together with the self-dual R-R field give anomalies that can be cancelled by the addition of a Chern-Simons term in the usual way. It is an amusing fact that whereas the cancellation of the $\operatorname{tr} R^{6}$ term in the type-I theory anomaly polynomial requires 496 Majorana-Weyl fermions to cancel the contributions of the gravitino and dilatino, the cancellation in this case takes place between the contributions of the self-dual R-R field and 496 Weyl fermions. It 
is clear that this is the right counting, since we know that in the type-IIB theory the gravitational anomaly contributions of the self-dual R-R field precisely cancel those of a pair of Majorana-Weyl gravitinos and dilatinos.

\subsection{Addition of brane-antibrane pairs}

Let us now consider adding $n$ D9-branes and $n$ anti-D9-branes to the $\mathrm{U}(32)$ theory. Clearly, this will give a theory that has $\mathrm{U}(32+n) \times \mathrm{U}(n)$ gauge symmetry. Also, it contains complex tachyon fields in the bifundamental representation. Like the typeIIB and type-I theories with added brane-antibrane pairs, which were analyzed in previous sections, we expect this theory to be perturbatively well behaved. Therefore the anomalies should cancel.

It is easy to figure out what the chiral fermions are in this case. The 99 open strings give left-handed Weyl fermions in the antisymmetric tensor representation of $\mathrm{U}(32+n)$, the $\overline{9} \overline{9}$ open strings give left-handed Weyl fermions in the symmetric tensor representation of $\mathrm{U}(n)$, and the $9 \overline{9}$ open strings give right-handed Weyl fermions in the bifundamental representation. The anomalies can be computed as usual, and one finds that the anomaly polynomial $I_{12}$ is proportional to the 12 -form piece of

$$
\left(\operatorname{Tr}_{A} e^{i F_{1}}+\operatorname{Tr}_{S} e^{i F_{2}}-\operatorname{tr} e^{i F_{1}} \operatorname{tr} e^{i F_{2}}\right) \hat{A}(R)+\frac{1}{8} L(R)
$$

where the subscript 1 refers to $\mathrm{U}(n+32)$ and the subscript 2 refers to $\mathrm{U}(n)$. The last term is the contribution of the self-dual R-R field. This expression looks just like $\left(\underline{\underline{B}} . \bar{z}_{1}^{\prime}\right)$, though now we are dealing with unitary groups instead of orthogonal groups. Just as in the previous case, the expression (14.1 $\left.{ }_{1}^{1}\right)$ can be recast in the form

$$
\frac{1}{2}\left(\left[\operatorname{Str} e^{i F}\right]^{2}-\operatorname{Str} e^{2 i F}\right) \hat{A}(R)+\frac{1}{8} L(R)
$$

where $\operatorname{Str} e^{i F}=\operatorname{tr} e^{i F_{1}}-\operatorname{tr} e^{i F_{2}}$. Again, the 12-form piece of this expression agrees with the 12 -form piece of $\frac{1}{2} Y^{2}$, where $Y$ is given by

$$
Y=\operatorname{Str} e^{i F} \sqrt{\hat{A}(R)}-32 \sqrt{L\left(\frac{R}{4}\right)} .
$$

One difference from the $\mathrm{SO}(32+n) \times \mathrm{SO}(n)$ type-I theory is that traces of odd powers of $F$ are now non zero. The anomaly can now be cancelled by the addition of a Chern-Simons term of the form $\mu \int C Y$. This formula is more like that of the IIB case in that $C=-i C_{0}+C_{2}+i C_{4}-C_{6}-i C_{8}$, with the same self-duality constraint as in that case. This example provides a pleasing mix of features of the type-I and type-IIB theories. Note that the relative U(1) gauge field eats the R-R scalar, just as in the type-IIB problem. The dependence on bifundamental tachyons could be added in the same way that it was added there. 


\section{Type I compactified on a smooth K3}

When the type-I superstring theory is compactified on a smooth K3, the requirement that $d H=\operatorname{tr} R^{2}-\operatorname{tr} F^{2}$ should be exact implies that there are 24 units of instanton number. This can be achieved by a combination of large instantons embedded in the $\mathrm{SO}(32)$ gauge group and small instantons (which are D5-branes) localized on the

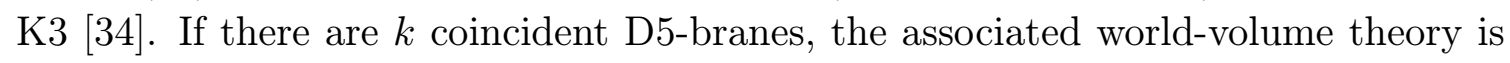
an $\operatorname{Sp}(k)$ gauge theory. In this case, the remaining $24-k$ units of instanton number must be embedded in the $\mathrm{SO}(32)$ gauge group, which breaks it to $\mathrm{SO}(8+k)$.

To be specific, we will focus on the case $k=24$, which gives the maximal symmetry group $\mathrm{SO}(32) \times \mathrm{Sp}(24)$. This theory has 32 D9-branes and 24 coincident D5-branes. The resulting $6 \mathrm{~d}$ theory has $\mathcal{N}=1$ supersymmetry ( 8 conserved supercharges). The massless fields consist of the supergravity multiplet, one tensor multiplet, vector multiplets for each of the gauge groups, and three classes of hypermultiplets. There are 20 hypermultiplets of gravitational origin, which are gauge singlets. The second class of hypermultiplets arises as zero modes of 55 strings. They belong to the antisymmetric tensor representation of the $\operatorname{Sp}(24)$ gauge group. This representation is reducible because it contains a symplectic traceless part and a singlet. The singlet provides the requisite Goldstone fermion. The third class of hypermultiplets arises as zero modes of 59 strings. They belong to the bifundamental representation, of course. These states actually belong to "half hypermultiplets", which is possible because the representation is pseudoreal.

The anomaly analysis of these systems and the other compactified systems we will consider could be analyzed from either a ten-dimensional or a six-dimensional viewpoint. In the former case the fivebranes would be described as localized defects embedded in ten dimensions and anomalies would be analyzed locally taking account of the phenomenon of "anomaly inflow". In the alternative six-dimensional viewpoint, one simply considers the effective six-dimensional theory that arises at length scales large compared to the compactification scale. This is the approach we will take in the following discussion. For example, in the particular example under consideration at the moment, altogether there are 244 more hypermultiplets than vector multiplets. This is the number required for the cancellation of the $\operatorname{tr} R^{4}$ piece of the anomaly 8 -form in $\mathcal{N}=16 \mathrm{~d}$ theories with one tensor multiplet [3.55].

The anomaly 8 -form for this $6 \mathrm{~d}$ theory was analyzed in detail in [i] was shown that it factorizes in the form

$$
I_{8}=-\frac{1}{16}\left(\operatorname{tr} R^{2}-\operatorname{tr} F_{9}^{2}\right)\left(\operatorname{tr} R^{2}+2 \operatorname{tr} F_{9}^{2}-2 \operatorname{tr} F_{5}^{2}\right) .
$$

Here $F_{9}$ refers to the $\mathrm{SO}(32)$ group and $F_{5}$ refers to the $\mathrm{Sp}(24)$ group. It follows that the gauge and Lorentz anomalies can be cancelled by adding a Chern-Simons term of the form $\int C_{2} Y_{4}$ and requiring that $C_{2}$ transform under Lorentz and gauge 
transformations in the usual fashion. Our goal in the remainder of this section is to explore how this analysis should be generalized when one allows for the addition of anti-D9-branes and anti-D5-branes.

We add $n$ D9-brane anti-D9-brane pairs and $m$ D5-brane anti-D5-brane pairs. Then the D9-branes and anti-D9-branes give the gauge group

$$
G_{9}=\mathrm{SO}(32+n) \times \mathrm{SO}(n)
$$

just as we found for the uncompactified theory in the preceding section. Taking all the D5-branes and all the anti-D5-branes to be coincident ${ }^{3}$ gives the gauge group

$$
G_{5}=\operatorname{Sp}(24+m) \times \operatorname{Sp}(m)
$$

This theory is unstable and non supersymmetric with tachyons, like the examples described in the preceding sections. As was done for those examples, we will identify the various chiral fermions, compute the associated anomaly polynomial, and deduce the Chern-Simons term of the effective theory.

Let us now consider the massless chiral fermions. We use the convention that fermions that have the same chirality as the supercharge (and hence of fermions in vector supermultiplets) are left-handed and contribute to the anomaly polynomial with a plus sign. Right-handed fermions, such as the ones in hypermultiplets, contribute with a minus sign. Here and in section ${ }^{\prime}, \overline{6}$, , handedness of fermions is understood in the six-dimensional sense.

Let us start with the zero modes of strings connecting the various 9-branes. Since the 99 spectrum is supersymmetric, these fermions are left-handed and belong to the adjoint representation. The $9 \overline{9}$ fermions are right-handed (as follows, for example, from the discussion in section $\left.3.2_{1}\right)$ and belong to the bifundamental representation. The $\overline{9} \overline{9}$ fermion zero modes are left-handed and belong to the symmetric representation of $\mathrm{SO}(n)$, just as we found previously in ten dimensions. Thus the Chern characters associated with these states give

$$
\operatorname{Tr} e^{i F_{9}}-\operatorname{tr} e^{i F_{9}} \operatorname{tr} e^{i F_{\overline{9}}}+\operatorname{Tr}_{S} e^{i F_{\overline{9}}}=\frac{1}{2}\left(\operatorname{Str} e^{i F_{9}}\right)^{2}-\frac{1}{2} \operatorname{Str} e^{2 i F_{9}} .
$$

As usual, Tr refers to the adjoint representation, tr to the fundamental representation, and $\operatorname{Tr}_{S}$ to the symmetric tensor representation.

Next let us consider the 55 spectrum. As before, the 55 strings give left-handed fermions in the adjoint representation and right-handed fermions in the antisymmetric tensor representation of the $\operatorname{Sp}(24+m)$ gauge group. For the $\overline{5} \overline{5}$ strings the chiralities are reversed: the adjoint fermions are right-handed and the antisymmetric

\footnotetext{
${ }^{3}$ It does not matter whether the D5-branes are coincident with the anti-D5-branes, since any massless fermions arising in this way would be non chiral. This has to be the case, because the two sets of branes can be separated without changing the gauge groups.
} 
tensor representation fermions are left-handed. ${ }^{4}$ As explained in a footnote above, there is no contribution from $5 \overline{5}$ strings. Altogether these states contribute

$$
\operatorname{Tr} e^{i F_{5}}-\operatorname{Tr} e^{i F_{5}}-\operatorname{Tr}_{A} e^{i F_{5}}+\operatorname{Tr}_{A} e^{i F_{5}}=\operatorname{Str} e^{2 i F_{5}}
$$

Finally, we have four classes of fermion zero modes for 59 strings. In each case we get a bifundamental representation, with the factor of $1 / 2$ explained earlier. The 95 and $\overline{9} \overline{5}$ fermion zero modes are right-handed whereas the $9 \overline{5}$ and $\overline{9} 5$ ones are left-handed. Thus these contributions give

$$
\begin{aligned}
-\frac{1}{2} & \operatorname{tr} e^{i F_{9}} \operatorname{tr} e^{i F_{5}}-\frac{1}{2} \operatorname{tr} e^{i F_{\overline{9}}} \operatorname{tr} e^{i F_{\overline{5}}}+\frac{1}{2} \operatorname{tr} e^{i F_{\overline{9}}} \operatorname{tr} e^{i F_{5}}+\frac{1}{2} \operatorname{tr} e^{i F_{9}} \operatorname{tr} e^{i F_{\overline{5}}}= \\
= & -\frac{1}{2} \operatorname{Str} e^{i F_{9}} \operatorname{Str} e^{i F_{5}} .
\end{aligned}
$$

Adding up the three sets of terms given above and expanding gives

$$
-224+6 \operatorname{Str} F_{5}^{2}-3 \operatorname{Str} F_{9}^{2}+\frac{1}{8}\left(\operatorname{Str} F_{9}^{2}\right)^{2}-\frac{1}{8} \operatorname{Str} F_{9}^{2} \operatorname{Str} F_{5}^{2}+\cdots .
$$

Including the gravitational contributions, one finds that the anomaly 8-form factorizes

$$
I_{8}=-\frac{1}{16} Y_{4}^{(1)} Y_{4}^{(2)}
$$

where

$$
Y_{4}^{(1)}=\operatorname{tr} R^{2}-\operatorname{Str} F_{9}^{2}
$$

and

$$
Y_{4}^{(2)}=\operatorname{tr} R^{2}+2 \operatorname{Str} F_{9}^{2}-2 \operatorname{Str} F_{5}^{2} .
$$

Given the result in the absence of the extra brane-antibrane pairs, this is the simplest outcome we could have expected.

It follows that the anomaly cancellation works as before. For example, if we define $Y_{4}^{(1)}=d \Omega_{3}$ and $\delta \Omega_{3}=d \Lambda_{2}$, then a Chern-Simons term of the form $\mu \int C_{2} Y_{4}^{(2)}$ can cancel the anomaly provided that we require $\delta C_{2}=\Lambda_{2}$. As usual, $H=d C_{2}-\Omega_{3}$ is then gauge invariant.

In a more symmetrical treatment $C_{2} Y_{4}^{(2)}$ would be replaced by $C_{2}^{+}\left(Y_{4}^{(2)}+Y_{4}^{(1)}\right)+$ $C_{2}^{-}\left(Y_{4}^{(2)}-Y_{4}^{(1)}\right)$, where $C_{2}^{ \pm}=\frac{1}{2}\left(C_{2} \pm \widetilde{C}_{2}\right)$. The self-dual part $C_{2}^{+}$belongs to the supergravity multiplet, and the anti-self-dual part $C_{2}^{-}$belongs to the tensor supermultiplet. (This separation is difficult to achieve covariantly, but that is a problem we face in all the examples with self-dual R-R forms.)

\footnotetext{
${ }^{4} \mathrm{~A} \overline{5}$-brane differs from a 5-brane by reflection of one of its worldvolume coordinates together with one of the normal coordinates, so the $\overline{5} \overline{5}$ spectrum can be deduced directly from the 55 spectrum. Alternatively, according to the reasoning in section $\overline{3} \cdot \overline{2}$, going from 55 to $\overline{5} \overline{5}$ should reverse the $\Omega$ projection in the $\mathrm{R}$ sector, exchanging the roles of symmetric and antisymmetric tensors of $\operatorname{Sp}(m)$ or in other words exchanging the adjoint representation with the antisymmetric tensor.
} 


\section{Type I compactified on a $\mathbb{T}^{4} / \mathbb{Z}_{2}$ orbifold}

\subsection{Review of the basic model}

The study of a particular compactification of the type-I theory on a $\mathbb{T}^{4} / \mathbb{Z}_{2}$ orbifold was explored in [3] The anomaly cancellation analysis of this model was subsequently carried out in [3. $\left.38_{-1}\right]$. This orbifold is a singular limit of a K3, so once again it is necessary to account for 24 units of instanton number. In the model of [ $[\overline{3} \bar{i}]$ one unit of instanton number is localized at each of the 16 fixed points of the orbifold and the remaining 8 units are realized as D5-branes. Equivalently, we can look at the R-R charge. Each orbifold point has a charge of $-3 / 2$ arising from the geometry and +1 from the embedded instanton for a total of $-1 / 2$. Even though none of the instantons is embedded in the $\mathrm{SO}(32)$ gauge group associated with the D9-branes (except as point instantons that do not break the gauge symmetry), the $\mathbb{Z}_{2}$ that is used to form the orbifold action acts on the gauge group breaking it to U(16). This group can be broken further by Wilson lines, as we will discuss.

Altogether the model has a gauge group of the form $G_{5} \times G_{9}$, where $G_{5}$ is associated with the D5-branes and $G_{9}$ is associated with the D9-branes. There is a rich set of possibilities for each. Those for the D5-branes can be understood geometrically, whereas those for the D9-branes correspond to various possibilities for the Wilson lines. Remarkably the two stories are isomorphic, and the full set of models that can be realized this way is invariant under T-duality. In other words, for every construction there is a dual construction for which the role of the D9-branes and the D5-branes is interchanged.

Let us recall the geometric description of the possible gauge groups $G_{5}$. Just as in the preceding section, when $k$ D5-branes coincide at a regular point of the the orbifold, the associated world-volume theory is an $\mathcal{N}=1 \operatorname{Sp}(k)$ gauge theory. However, when fivebranes approach an orbifold point, the mirror images come into play and the group is enhanced to $\mathrm{U}(2 k)$. Thus, for example, if all eight of them are on the same orbifold point this gives $G_{5}=\mathrm{U}(16)$. Another interesting fact is that half fivebranes can attach to orbifold points, so that unitary groups of odd rank are also possible. Only an integral number of fivebranes can move off of an orbifold point. One extreme case is to attach a half fivebrane to each of the orbifold points giving the group $G_{5}=\mathrm{U}(1)^{16}$. This configuration is special, in that it is the only one that cancels the R-R charge locally. However, it is not necessary to do that. Thus there are a number of topologically distinct sectors characterized by the number (and locations) of orbifold points that have a half fivebrane attached to them. As required by T-duality, there are corresponding statements that could be made about the possible configurations of Wilson lines and their implications for $G_{9}$. According

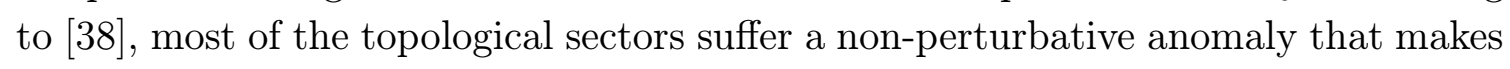
them unacceptable, even though they are all consistent perturbatively. The rule is that the number of orbifold points with attached half fivebranes must be either 0 , 
8, or 16. Since one also has the corresponding restriction for the Wilson lines, there are 9 different topological sectors that are allowed, but only 6 of them are distinct when T-duality is taken into account.

We will review how the anomaly analysis works for this class of models. Later we will generalize the results to allow for the addition of anti-D5-branes and antiD9-branes. For describing the analysis it is convenient to assign all the fivebranes to orbifold points, so that $G_{5}=\prod_{1}^{16} \mathrm{U}\left(m_{i}\right)$, where $\sum_{1}^{16} m_{i}=16$. Note that we include all the orbifold points in the sum, since we allow the $m_{i}$ 's to be zero. This helps to keep the notation relatively simple. Situations in which there are fivebranes in the bulk correspond to a simple Higgsing of this class of models. On the other hand, the anomaly analysis of these models is more subtle than those with only fivebranes in the bulk, because of the U(1) factors. Therefore by considering the model with this choice of $G_{5}$ and $G_{9}=\prod_{1}^{16} \mathrm{U}\left(n_{i}\right)$, where $\sum_{1}^{16} n_{i}=16$, we are really taking account of all interesting cases.

\subsection{The massless spectrum}

As in the preceding section, the massless gauge singlet fields, associated to zero modes of closed strings, consist of the supergravity multiplet, a tensor multiplet, and 20 hypermultiplets. One significant fact is that 16 of these hypermultiplets are associated to the 16 orbifold points. One of the scalars in each of the hypermultiplets belongs to the R-R sector. The corresponding $16 \mathrm{R}-\mathrm{R}$ scalar fields play an important role in the anomaly analysis.

Let us now consider the part of the massless spectrum that arises as zero modes of open strings. Most of it is pretty obvious. Consider the $5_{i} 5_{j}$ open string spectrum first. It is clear that there is no contribution from open strings connecting fivebranes at one orbifold point to ones at a different orbifold point, since they are spatially separated. So we need only consider the case $i=j$. These give vector supermultiplets for each of the $\mathrm{U}\left(m_{i}\right)$ groups. In addition, they also give two hypermultiplets belonging to antisymmetric tensor representations. The spectrum of 99 strings is completely analogous, as required by T-duality.

We now turn to the spectrum of zero modes of $5_{i} 9_{j}$ open strings. This spectrum,

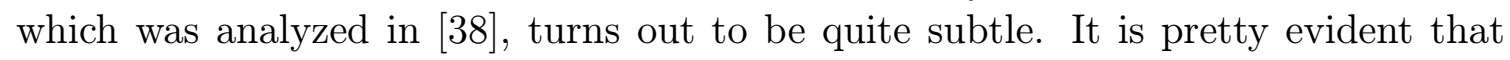
one expects each of the possible 59 open strings to give a massless hypermultiplet in the bifundamental representation. The subtlety is that there are two possibilities $\left(m_{i}, n_{j}\right)$ and $\left(m_{i}, \bar{n}_{j}\right) .{ }^{5}$ For any given pair $i, j$, the distinction between these two cases is a matter of convention, as it can be reversed by complex conjugation of one of the groups. However, in general, there are more distinct pairings than there are unitary groups, so this distinction is not entirely convention. Therefore let us distinguish the

\footnotetext{
${ }^{5}$ Recall that a hypermultiplet contains the complex conjugate, so $\left(m_{i}, n_{j}\right)$ is the same as $\left(\bar{m}_{i}, \bar{n}_{j}\right)$ and $\left(m_{i}, \bar{n}_{j}\right)$ is the same as $\left(\bar{m}_{i}, n_{j}\right)$.
} 
two possibilities by a parameter $w_{i j}$ that takes the value +1 for values of $i$ and $j$ such that the 59 open strings transform as $\left(m_{i}, n_{j}\right)$ and the value -1 in the second case. This matrix of parameters satisfies certain properties that we will describe later.

\subsection{Anomaly analysis}

Let us now focus on the Chern characters that enter in the anomaly analysis. The $9_{i} 9_{i}$ chiral fermions give

$$
\operatorname{Tr} e^{i F_{9 i}}-2 \operatorname{Tr}_{A} e^{i F_{9 i}}=n_{i}-2 \operatorname{tr} F_{9 i}^{2}+2\left(\operatorname{tr} F_{9 i}\right)^{2}+\frac{2}{3} \operatorname{tr} F_{9 i}^{4}-\frac{2}{3} \operatorname{tr} F_{9 i} \operatorname{tr} F_{9 i}^{3}+\cdots .
$$

Similarly, the $5_{i} 5_{i}$ chiral fermions give

$$
\operatorname{Tr} e^{i F_{5 i}}-2 \operatorname{Tr}_{A} e^{i F_{5 i}}=m_{i}-2 \operatorname{tr} F_{5 i}^{2}+2\left(\operatorname{tr} F_{5 i}\right)^{2}+\frac{2}{3} \operatorname{tr} F_{5 i}^{4}-\frac{2}{3} \operatorname{tr} F_{5 i} \operatorname{tr} F_{5 i}^{3}+\cdots .
$$

The $5_{i} 9_{j}$ chiral fermions, with the rule described above, give

$$
\begin{aligned}
-\operatorname{tr} e^{i F_{5 i}} \operatorname{tr} e^{i w_{i j} F_{9 j}}= & -m_{i} n_{j}+\frac{1}{2} m_{i} \operatorname{tr} F_{9 j}^{2}+\frac{1}{2} n_{j} \operatorname{tr} F_{5 i}^{2}+w_{i j} \operatorname{tr} F_{5 i} \operatorname{tr} F_{9 j}- \\
& -\frac{1}{24} m_{i} \operatorname{tr} F_{9 j}^{4}-\frac{1}{24} n_{j} \operatorname{tr} F_{5 i}^{4}-\frac{1}{4} \operatorname{tr} F_{5 i}^{2} \operatorname{tr} F_{9 j}^{2}- \\
& -\frac{1}{6} w_{i j} \operatorname{tr} F_{5 i}^{3} \operatorname{tr} F_{9 j}-\frac{1}{6} w_{i j} \operatorname{tr} F_{5 i} \operatorname{tr} F_{9 j}^{3}+\cdots
\end{aligned}
$$

Combining all of the above give

$$
\begin{aligned}
-224 & +6 \sum_{i} \operatorname{tr} F_{9 i}^{2}+6 \sum_{i} \operatorname{tr} F_{5 i}^{2}-\frac{1}{4} \sum_{i} \operatorname{tr} F_{5 i}^{2} \sum_{j} \operatorname{tr} F_{9 j}^{2}+2 \sum_{i}\left(\operatorname{tr} F_{9 i}\right)^{2}+ \\
& +2 \sum_{i}\left(\operatorname{tr} F_{5 i}\right)^{2}+\sum_{i j} w_{i j} \operatorname{tr} F_{5 i} \operatorname{tr} F_{9 j}-\frac{2}{3} \sum_{i} \operatorname{tr} F_{9 i} \operatorname{tr} F_{9 i}^{3}- \\
& -\frac{2}{3} \sum_{i} \operatorname{tr} F_{5 i} \operatorname{tr} F_{5 i}^{3}-\frac{1}{6} \sum_{i j} w_{i j} \operatorname{tr} F_{5 i}^{3} \operatorname{tr} F_{9 j}-\frac{1}{6} \sum_{i j} w_{i j} \operatorname{tr} F_{5 i} \operatorname{tr} F_{9 j}^{3} .
\end{aligned}
$$

Multiplying this by $\hat{A}(R)$, adding the closed-string contribution, and extracting the 8-form gives

$$
\begin{aligned}
I_{8}= & -\frac{1}{16}\left(\operatorname{tr} R^{2}\right)^{2}+\frac{1}{8} \operatorname{tr} R^{2}\left(\sum_{i} \operatorname{tr} F_{9 i}^{2}+\sum_{i} \operatorname{tr} F_{5 i}^{2}\right)-\frac{1}{4} \sum_{i} \operatorname{tr} F_{5 i}^{2} \sum_{j} \operatorname{tr} F_{9 j}^{2}+ \\
& +\frac{1}{24} \operatorname{tr} R^{2}\left(\sum_{i}\left(\operatorname{tr} F_{9 i}\right)^{2}+\sum_{i}\left(\operatorname{tr} F_{5 i}\right)^{2}+\frac{1}{2} \sum_{i j} w_{i j} \operatorname{tr} F_{5 i} \operatorname{tr} F_{9 j}\right)+ \\
& -\frac{2}{3} \sum_{i} \operatorname{tr} F_{9 i} \operatorname{tr} F_{9 i}^{3}-\frac{2}{3} \sum_{i} \operatorname{tr} F_{5 i} \operatorname{tr} F_{5 i}^{3}-\frac{1}{6} \sum_{i j} w_{i j} \operatorname{tr} F_{5 i}^{3} \operatorname{tr} F_{9 j}- \\
& -\frac{1}{6} \sum_{i j} w_{i j} \operatorname{tr} F_{5 i} \operatorname{tr} F_{9 j}^{3} .
\end{aligned}
$$


The key identity [3. achieve anomaly cancellation is

$$
\sum_{i=1}^{16} w_{i j} w_{i k}=16 \delta_{j k} .
$$

Using this, we can recast $I_{8}$ in the factorized form

$$
I_{8}=-\frac{1}{4} Y_{4}^{(5)} Y_{4}^{(9)}-\frac{2}{3} \sum_{i=1}^{16} Y_{2 i} Y_{6 i}
$$

where

$$
\begin{aligned}
Y_{4}^{(5)} & =\frac{1}{2} \operatorname{tr} R^{2}-\sum_{i} \operatorname{tr} F_{5 i}^{2}, \\
Y_{4}^{(9)} & =\frac{1}{2} \operatorname{tr} R^{2}-\sum_{i} \operatorname{tr} F_{9 i}^{2}, \\
Y_{2 i} & =\operatorname{tr} F_{5 i}+\frac{1}{4} \sum_{j} w_{i j} \operatorname{tr} F_{9 j}, \\
Y_{6 i} & =\operatorname{tr} F_{5 i}^{3}+\frac{1}{4} \sum_{j} w_{i j} \operatorname{tr} F_{9 j}^{3}-\frac{1}{16} \operatorname{tr} R^{2} Y_{2 i} .
\end{aligned}
$$

Thus anomaly cancellation is achieved by adding Chern-Simons terms of the form

$$
\mu \int\left(C_{2} Y_{4}^{(9)}+\sum_{i=1}^{16} C_{0 i} Y_{6 i}\right) .
$$

Here, $C_{0 i}$ are the $16 \mathrm{R}-\mathrm{R}$ scalars that were pointed out earlier. This completes the review of results from [3- $3 \overline{8}$. Now, we are ready to consider adding additional braneantibrane pairs.

\subsection{Addition of brane-antibrane pairs}

Let us now consider adding additional brane-antibrane pairs. As before we will only consider the case when all the D5-branes and anti-D5-branes are located at the orbifold points so that the gauge group is a product of unitary groups. Letting $m_{i}$ denote the number of half-D5-branes and $\widetilde{m}_{i}$ the number of half anti-D5-branes at the $i$-th orbifold point, the gauge group is

$$
G_{5}=\prod_{i=1}^{16} \mathrm{U}\left(m_{i}\right) \times \mathrm{U}\left(\widetilde{m}_{i}\right) .
$$

Each of the $m_{i}$ and $\widetilde{m}_{i}$ is allowed to be either zero or a positive integer. Since the total fivebrane charge must be 8 , the only perturbative restriction is

$$
\sum_{i=1}^{16}\left(m_{i}-\widetilde{m}_{i}\right)=16 .
$$


The D9-branes are treated in similar manner, so that

$$
G_{9}=\prod_{i=1}^{16} \mathrm{U}\left(n_{i}\right) \times \mathrm{U}\left(\widetilde{n}_{i}\right)
$$

where

$$
\sum_{i=1}^{16}\left(n_{i}-\widetilde{n}_{i}\right)=16 .
$$

Let us now consider the spectrum of chiral fermions arising from open-string zero modes [30i]. As before the $9_{i} 9_{i}$ and $5_{i} 5_{i}$ open strings give left-handed fermions in the adjoint representation and two copies of right-handed fermions in the antisymmetric tensor representation. The $\overline{9}_{i} \overline{9}_{i}$ and $\overline{5}_{i} \overline{5}_{i}$ open strings give left-handed fermions in the adjoint representation and two copies of right-handed fermions in the symmetric tensor representation. The $9_{i} 5_{j}$ and $\overline{9}_{i} \overline{5}_{j}$ open strings give right-handed fermions in a bifundamental representation, whereas the $9_{i} \overline{5}_{j}$ and $\overline{9}_{i} 5_{j}$ opens strings give left-handed fermions in a bifundamental representation. In each of these four cases the issue of whether one has (fundamental, fundamental) or (fundamental, antifundamental) is described by the parameters $w_{i j}$ introduced earlier.

At this point, it is easy to verify that the number of right-handed fermions exceeds the number of left-handed ones by 244, as required. However, this is not yet the whole story. There are still $9_{i} \overline{9}_{i}$ and $5_{i} \overline{5}_{i}$ open-string zero modes to be taken into account. They do provide additional chiral content, even though they give an equal number of left-handed and right-handed fermions. The correct rule in each case is that they give (fundamental, fundamental) and (antifundamental, antifundamental) left-handed fermions and (fundamental, antifundamental) and (antifundamental, fundamental) right-handed fermions. The contribution of this set of states to the Chern character term in the anomaly is

$$
\left(\operatorname{tr} e^{i F_{9 i}}-\operatorname{tr} e^{-i F_{9 i}}\right)\left(\operatorname{tr} e^{i F_{\overline{9}} i}-\operatorname{tr} e^{-i F_{\overline{9}} i}\right)=-4 \operatorname{tr} \sin F_{9 i} \operatorname{tr} \sin F_{\overline{9} i} .
$$

Taking all of the above into account, it is straightforward to recompute the anomaly 8-form $I_{8}$. The answer turns out to be just what one might have guessed. Namely, $I_{8}$ is still given by eq. ('(6...7.

$$
\begin{aligned}
Y_{4}^{(5)} & =\frac{1}{2} \operatorname{tr} R^{2}-\sum_{i} \operatorname{Str} F_{5 i}^{2}, \\
Y_{4}^{(9)} & =\frac{1}{2} \operatorname{tr} R^{2}-\sum_{i} \operatorname{Str} F_{9 i}^{2}, \\
Y_{2 i} & =\operatorname{Str} F_{5 i}+\frac{1}{4} \sum_{j} w_{i j} \operatorname{Str} F_{9 j}, \\
Y_{6 i} & =\operatorname{Str} F_{5 i}^{3}+\frac{1}{4} \sum_{j} w_{i j} \operatorname{Str} F_{9 j}^{3}-\frac{1}{16} \operatorname{tr} R^{2} Y_{2 i} .
\end{aligned}
$$


Thus the anomaly cancellation works as before with the substitution of supertraces for traces. This seems to be the general rule.

\section{Acknowledgments}

Part of this material was presented in the lecture given by JHS at the "Davidfest" Conference held at the ITP in Santa Barbara on March 2-3, 2001. This work was supported in part by the U.S. Dept. of Energy under Grant No. DE-FG03-92ER40701 and by the Caltech Discovery Fund.

\section{References}

[1] M. Srednicki, IIB or not IIB, 'J. High Energy_Phys o8 (1998) 0051 [hep-th/9807138].

[2] M.B. Green, J.A. Harvey and G. Moore, I-brane inflow and anomalous couplings on D-branes, 'Class. and Quant. Grav. 14 1997$)$ 47' [hep-th/9605033].

[3] D.S. Freed and M.J. Hopkins, On Ramond-Ramond fields and K-theory, iJ. i. ergy Phys. 05

[4] G. Moore and E. Witten, Self-duality, Ramond-Ramond fields and K-theory, ${ }^{\prime}$. : E

[5] T. Yoneya, Spontaneously broken space-time supersymmetry in open string theory without GSO projection, Nucl. Phys. B $\mathbf{5 7 6}(2000) 219$ i [hep-th/9912255j.

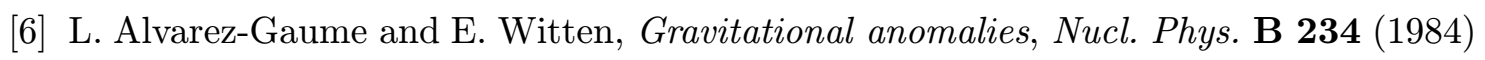
-

[7] M.B. Green and J.H. Schwarz, Anomaly cancellation in supersymmetric $D=10$ gauge

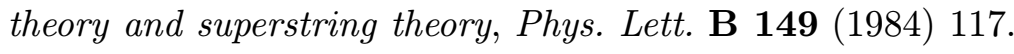

[8] A. Sagnotti, A note on the Green-Schwarz mechanism in open string theories, ${ }_{1}^{\bar{P}} \bar{h} \bar{y}$ s. L - Lett. B 294 (1992) 196: [hep-th/9210127i].

[9] C. Kennedy and A. Wilkins, Ramond-Ramond couplings on brane-antibrane systems,

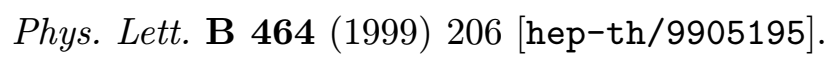

[10] P. Kraus and F. Larsen, Boundary string field theory of the DD system, hep-th/0012198.

[11] T. Takayanagi, S. Terashima and T. Uesugi, Brane-antibrane action from boundary string field theory, 'J. High Energy Phys.

[12] D. Quillen, Superconnections and the Chern character, Topology 24 (1985) 89.

[13] A. Sen, Tachyon condensation on the brane antibrane system, iJ. : 
[14] S. Sugimoto, Anomaly cancellations in type $i$ d9-d9-bar system and the usp(32) string

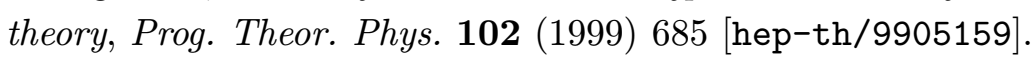

[15] M. Berkooz and A. Kapustin, A comment on nonsupersymmetric fixed points and

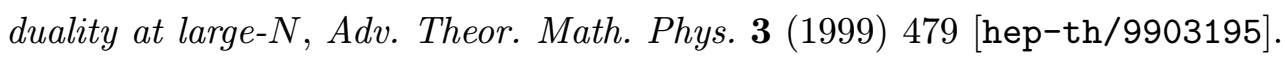

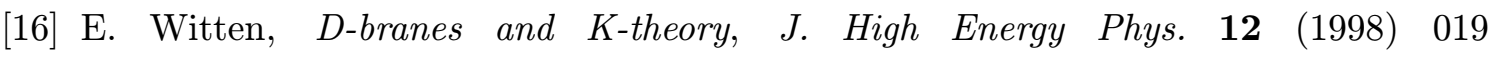
[hep-th/9810188i].

[17] J. Polchinski, String theory, vol. 2 Cambridge University Press, 1998.

[18] J.F. Morales, C.A. Scrucca and M. Serone, Anomalous couplings for D-branes and

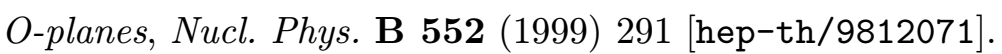

[19] C.A. Scrucca and M. Serone, Anomaly inflow and $\mathrm{rr}$ anomalous couplings, hep-th/9911223.

[20] J. Bogdan Stefanski, Gravitational couplings of D-branes and O-planes, : - . -

[21] E. Dudas and J. Mourad, Brane solutions in strings with broken supersymmetry and

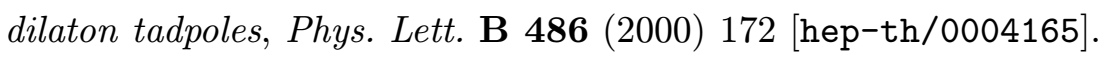

[22] R. Blumenhagen and A. Font, Dilaton tadpoles, warped geometries and large ex-

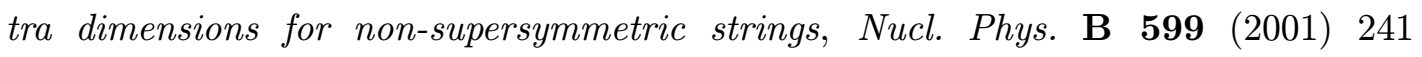
[hep-th/0011269i].

[23] E. Dudas and J. Mourad, Consistent gravitino couplings in non-supersymmetric strings, hep-th/0012071.

[24] L.J. Dixon and J.A. Harvey, String theories in ten-dimensions without space-time supersymmetry, №cl. Phys. B $\mathbf{2} 74$ (1986) 93 .

[25] L. Alvarez-Gaume, P. Ginsparg, G. Moore and C. Vafa, An $O(16) \times O(16)$ heterotic string, iPhys. Lett.

[26] A. Sagnotti, Some properties of open string theories, hep-th/95090801.

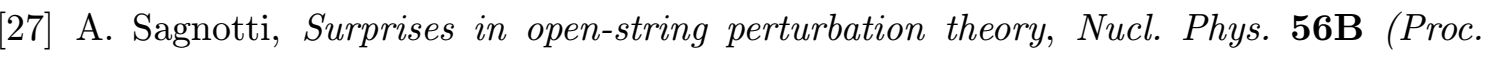

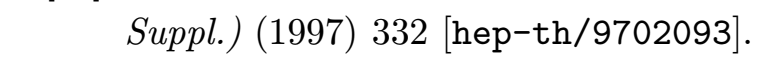

[28] A. Sagnotti, Open-string models with broken supersymmetry, 'Nucl. Phys. $\mathbf{8} \mathbf{8}$ (P roc.'

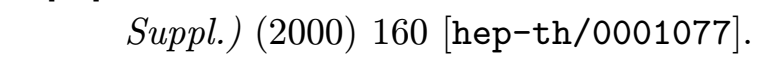

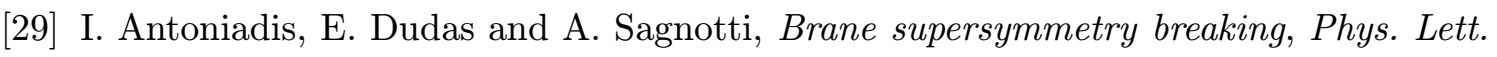
(1) -

[30] G. Aldazabal and A.M. Uranga, Tachyon-free non-supersymmetric type-IIB ori-

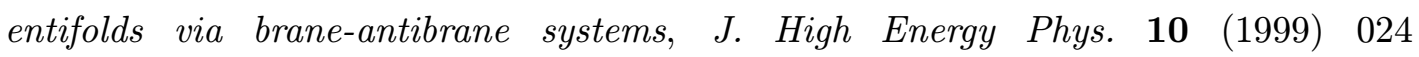
[hep-th/99080720']. 
[31] N. Seiberg and E. Witten, Spin structures in string theory, Nucl. Phys. B - -

[32] R. Blumenhagen, A. Font and D. Lust, Tachyon-free orientifolds of type 0b strings in various dimensions, iNucl. Phys. B 558 (1999) 159i [hep-th/9904069].

[33] E. Dudas and J. Mourad, D-branes in non-tachyonic OB orientifolds, '

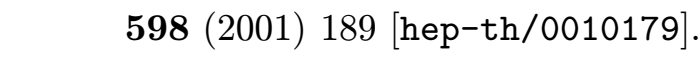

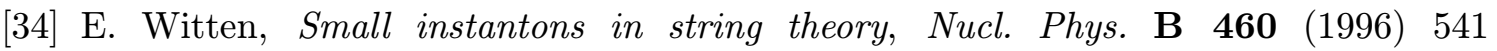
[hep-th/9511030i,

[35] M.B. Green, J.H. Schwarz and P.C. West, Anomaly free chiral theories in sixdimensions, 'Nucl.

[36] J.H. Schwarz, Anomaly-free supersymmetric models in six dimensions, "Phys. Letett. :

[37] E.G. Gimon and J. Polchinski, Consistency conditions for orientifolds and D-

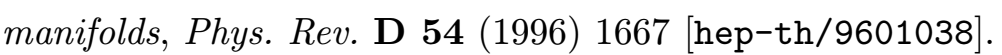

[38] M. Berkooz, R. G. Leigh, J. Polchinski, J. H. Schwarz, N. Seiberg and E. Witten,

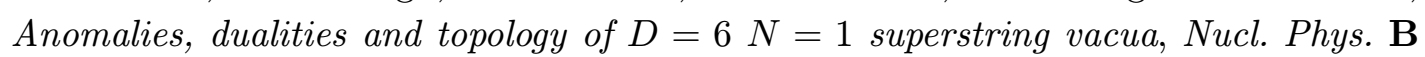
$47 \overline{5}(\overline{1} 9 \overline{6})$ 11 $\overline{1}_{1}^{1}$ [hep-th/9605184. 